\section{Transmembrane protein of SIV}

SIR-We would like to add to the observations on the transmembrane protein (TMP) of simian immunodeficiency virus (SIV) reported by Hirsch et al. in a previous scientific correspondence ${ }^{1}$. Immuno-

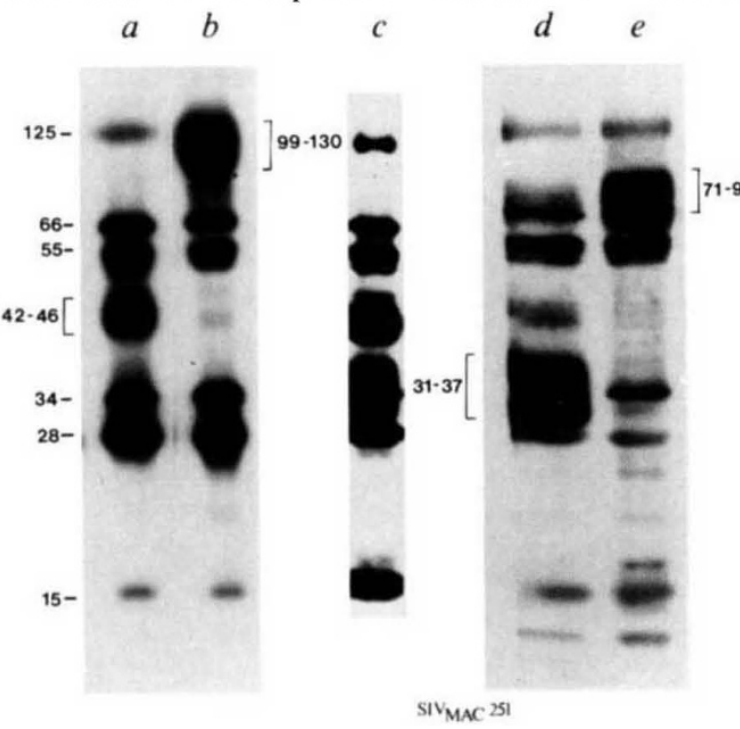

FIG. 1 Virus was purified by ultracentrifugation and chromatography on Sepharose $4 \mathrm{~b}$ from cultures of infected C8166 cells. The cultures were passed every 3 or 4 days by feeding with uninfected cells in a ratio of $1: 3$. Tracks $a, b$, pass 6 ; track $c$, pass 14 ; tracks $d, e$, pass 18 . Tracks $b, e$ were applied in sample buffer without SDS. Virus antigen was electrophoresed on $12.5 \%$ polyacrylamide gels containing SDS and proteins detected by probing with serum from an SIVinfected monkey followed by ${ }^{125}$ I-labelled Protein A. Relative molecular masses $(K)$ were estimated from standards.
FIG. 2 Comparison of the DNA 215 and amino-acid sequence of a portion of transmembrane region 8771 of the env gene of $\operatorname{SIV}_{\text {Mac. DNA }}$. $32 \mathrm{H}$ was isolated from infected (1ow pass) C8166 cells at low or high passage. PCR-amplified TMP-coding region was cloned and sequenced by standard procedures and the results compared to those published for two molecular clones of SIV (ref. 6). $\boldsymbol{\Delta}$, Nucleotide change; aminoacid change, $\star \star \star$, termination codon.

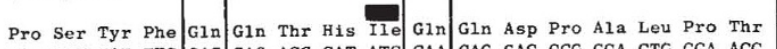
Pro Ser Tyr Phe Gln Gln Thr His Tle Gin Gln Asp Pro Ala Leu Pro Thr $32 \mathrm{H}$
(high pass) Pro Ser Tyr Phe Gln Gin Thr His Ile *** Gln Asp Pro Ala Leu Pro Thr Pro ser Tyr Phe Gin Gin Thr His ine ${ }^{* * *}$ Gin Asp Pro Ala leu pro Thr $\operatorname{SIV}_{\text {MAC }} 239$

Pro Ser Tyr Phe Gin Gln Thr His Ile Gln Gln Asp Pro Ala Leu Pro Thr

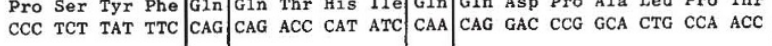

blot analysis of purified virus from a noncloned pool of infectious SIV $_{\text {MAC }}$, isolated from a rhesus macaque infected with SIV $_{\text {MAC 251 }}$ (virus supplied by R. Desrosiers) demonstrated the presence of a diffuse protein of relative molecular mass $42-$ 46,000 $\left(M_{\mathrm{r}} 42-46 \mathrm{~K}\right)$ (Fig. $\left.1 a\right)$. This virus pool, produced by co-cultivation of monkey peripheral-blood lymphocytes with phytohaemagglutinin-stimulated human cord-blood lymphocytes for 17 days followed by growth on the human C8166 T-cell line for 20 days, induces fatal AIDS-like disease in rhesus macaques ${ }^{2}$ and is intended for use as a vaccine challenge stock. Further passage of this virus in C8166 cells resulted first in a population displaying proteins with $M_{\mathrm{r}}$ of both $42-46 \mathrm{~K}$ and $31-37 \mathrm{~K}$ (Fig. 1c) and later a predominance of $31-37 \mathrm{~K}$ material (Fig. $1 d$ ). Nucleotide sequencing of polymerase chain reaction (PCR) amplified proviral DNA corresponding to the TMP-coding region of the SIV env gene revealed the presence of an in-frame stop codon in all six clones derived from the more highly for the presence of oligomeric TMP. It has been reported for HIV-1, that heat stable oligomers of TMP may be detected by immunoblotting when SDS concentrations are reduced in the PAGE sample buffer ${ }^{3}$. Both full-length (Fig. 1 $a, b$ ) and truncated (Fig. 1d,e) SIV TMP were found to form such oligomers; the estimated relative molecular masses indicate, in both cases, that these complexes are composed of dimers or trimers or both.

An experimental whole-inactivatedvirus vaccine, currently under trial in rhesus and cynomologus macaques contains full-length and truncated TMP (Fig. 1c). Because infected animals exhibit antibody responses to peptides from the cytoplasmic C-terminal domain of SIV

1. Hirsch, V. et al. Nature 341, 573-574 (1989) TNO Meeting on Animal Models in AIDS (ed. M. Horzinek) (Elsevier/North Holland Biomedical, in the press).

3. Pinter, A et al. J. Virol. 63, 2674-2679 (1989).

4. Franchini, G. et al. AIDS Res. hum. Retrovir. 4, 251-258 (1988).

5. Chakrabarti, L., Emerman, M., Tiollais, P. \& Sonigo, P. J. Virol. 63, 4395-4403 (1989).

6. Naidu, Y.M. et al. J. Virol. 62, 4691-4696 (1988)
TMP (ref. 4) and this region modulates growth properties of the virus, at least in vitro ${ }^{5}$, we believe inclusion of this region in putative vaccines to be important.

M. P. CRanage

Division of Pathology, PHLS Centre for Applied Microbiology \& Research, Porton Down, Salisbury SP4 OJG, UK

N. ALMOND

A. JENKINS

P. A. KITCHIN

WHO AIDS Collaborating Centre,

National Institute for Biological

Standards and Control, South Mimms, Potters Bar, Herts EN6 3QG, UK

\section{Genetic code origins}

SIR-We wish to point out a remarkable circumstance associated with the recent characterization of the guanosine/argininebinding site in Tetrahymena intronic RNA.

F. Michel and colleagues ${ }^{1}$, elsewhere in this issue, show that nucleotide pair G264.C311 contributes to the specificity of this part of the catalytic site, and suggest that G264 is hydrogen-bonded to the guanosine/arginine ligands. The remarkable circumstance is that this sidechainand stereo-selective arginine site $^{2}$ is formed by the conjunction of two arginineencoding triplets, AGA and CGG:

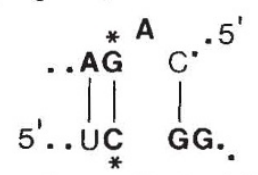

The base pairs marked with vertical bars form the end of the conserved helix, conventionally called $\mathrm{P} 7$, and the arginine codons are in bold type. The critical base pair is between the asterisks.

Arginine has six possible codons, and

2. Cranage, M.P. et al. in Proceedings of the Internationa

arginine codons comprise 6.1 per cent of the contiguous triplets in the Tetrahymena intron. Given an arbitrary choice of two nucleotides, the probability that both lie within a codon for arginine (in any phase) is 0.029 . The above observation would not, therefore, be sufficiently rare in itself to imply more than coincidence. In fact, the CGG codon itself is easily dismissed as coincidental. It is not phylogenetically conserved, instead being rather rare among the 66 tabulated group I intron sequences $^{3}$.

The AGA codon, however, is different. Although the P7 region is well conserved, there is considerable variation at the AGA codon. Among the 66 group I sequences there are 32 AGA codons (48 per cent), 31 CGA codons (47 per cent), and 3 AGG codons ( 5 per cent) occurring at the same positions in the sequence. However, these three sets of triplets all encode arginine. Thus, although there is variation on both sides of the completely 